\title{
Coordination Mechanism and Negotiation Model to Resolve Conflict of Communication Virtual Service in Information Network Corporation
}

\author{
Zhang Tao ${ }^{1}$ and Zhang Zhenji ${ }^{2}$ \\ ${ }^{1}$ China Center for Industrial Security Research \\ State Administration of Press, Publication, Radio, Film and Television \\ Beijing, China, 100866 \\ ${ }^{2}$ School of Economics and Management \\ Beijing JiaoTong University, Beijing, China, 100044 \\ ljantao123@hotmail.com, ${ }^{2}$ zhjzhang@bjtu.edu.cn
}

\begin{abstract}
Information Network Communications Group has introduced virtual services to seize the market, the communication channel resources appear uneven conflict. Based on characteristics of complex systems provide virtual network services when the information network of the Group, with the structural model and Multi-Agent ontology descriptions, give full play to the advantages of multi- Agent system in distributed computing and collaboration, combined with the company features a modern organizational structure, analysis its public resources collaborative model of conflict resolution, the establishment of a public resource conflict resolution strategies based on dynamic compensation.
\end{abstract}

Keywords: Multi-Agent, Resource conflict, Coordination mechanism, Negotiation strategy

\section{Introduction}

With the rapid development of information network market, the information network companies launched various types of communication services to seize the market. However, the needs of customers are hard to grasp experience according to the actual market experience. Thus, it was found that customers' requirement was difficult to realize because the virtual services can't correspond to the individual needs. With the business development, there has been the systems of various homogeneous virtual service accumulation, the limited audience of virtual services customer base, as well as the high cost of maintaining the size of pre-and post service issues such as lack of planning, a great impact on the core competitiveness of enterprise communications.

Group subsidiaries under management, business process operations is a multi-part interaction, dynamic open-ended questions multiple interacting factors, its subject is the role of the Group and its subsidiaries in the business life cycle division, based on the Group - a subsidiary organizational model, with the expansion of the diverse needs of virtual services, there is a conflict occurs when using public resources. With structural model and Multi-Agent ontology descriptions, give full play to the advantages of multi- Agent system in distributed computing and collaboration to explore public resource conflicts based on dynamic compensation digestion modern enterprise Group Company of public resources, organizational structure and collaborative conflict resolution mode strategy. There have appeared such problems as following: 
Various homogeneous virtual services have been piled up.

The customers covered by virtual services are limited.

The maintaining cost of a great number of service businesses is high.

The pre- and post information issues are lack of planning. These unexpected problems proposed the core competiveness of communication enterprises. The business procession is an opening dynamic question with multi interacting factors and multi-part interaction under Group-Subsidiary management. The subject of the question is the role niche of the group and its subsidiaries in the business cycle based on the organizational model. With the expansion of various types of virtual services, the conflicts occur when using public resources. According to the structural model and multi-agent ontology descriptions, the Groupsubsidiary structure might compensate and digest the public resource conflict with the help of multi-agent system in distributed computing and collaboration.

\section{Conflict Resolution Strategy Review of MAS}

Conflict resolution is one of the important issues in complex adaptive system (CAS) field. Currently, the conflict resolution has been studied extensively by many researchers in diverse fields. Many methods have been proposed as: Backtracking, Constraint Relaxation, Negotiation, etc., Negotiation strategy to resolute conflict is a new issue in recent years based on the research fruit of artificial intelligence (AI) field. This strategy is considered to be worth implored deeply by many experts. Negotiation is also one of the most commonly used methods when human solving conflict problems. When people have different views of things, views or serious disagreement, people would like to settle their differences through negotiation.

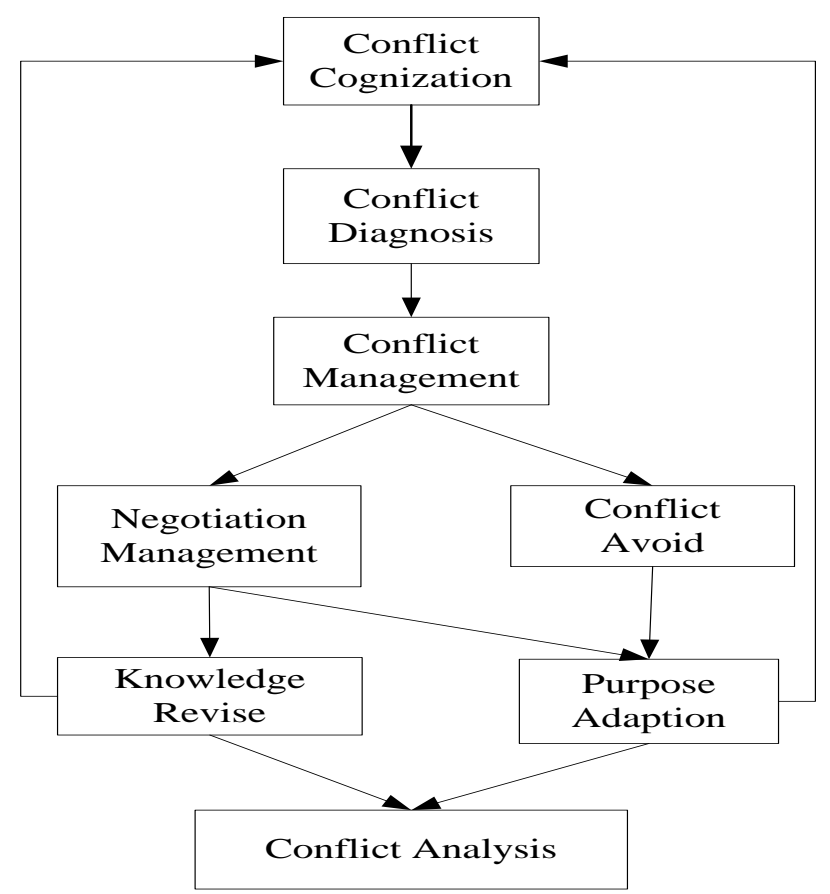

Figure 1. The Action Model of MAS Conflict Control 


\subsection{Backtracking}

The basic idea of backtracking method:

When conflict occurs, the root causes of the conflict should be first found. During the backtracking, the key nodes should also be acknowledged to help the following modify procession. By backtracking, the incompatible environment and the corresponding structural model should be modified to eliminate the conflict. Backtracking method includes order backtracking and related backtracking. Order backtracking considers the nearest node with a large amount of backtracking. Related backtracking considers mainly the related node which is the most interrelated nodes, regardless of the independent node with higher efficiency.

\subsection{Constraint Relaxation}

The basic idea of constraint relaxation:

All members in the collaborative environment established the various constraints and interdependence mechanism to constrain the behavior. When conflict occurs, the members would relax some constraints or ignored some less important constraints to achieve the purpose of conflict resolution. However, the relaxation of constraints or ignore constraints tend to make some of collaborative goals and ways of the individual change, thus the tasks can't be completed on time. Some members may also refuse to accept constraint relaxation, so the conflict would fail to be resolved. Constraint relaxation has certain limitations when dealing with the conflict in the corporations. Constraint relaxation usually is suitable for the internal conflict resolution in the team or the low degree conflict.

\subsection{Negotiation}

Negotiation is a dynamic process for the agents to discuss the issue to reach a consensus. To establish the negotiation model, the negotiation process of two agents is a limited cycle which can be designed as the following steps.

1) Agent 1 makes a proposal to Agent 2;

2) Agent 2 responded to Agent 1 on the proposal. If satisfied then accept it and the negotiation terminated. Otherwise agent 2 put forward a new proposal to Agent 1;

3) Agent 1 responded to Agent 2 on the new proposal. If satisfied then accept it and the negotiation terminated. Otherwise agent 1 make another new proposal to Agent 2;

4) Repeat Step 2 until both are satisfied and reaching the appointed time.

The negotiation strategy of conflict resolution stressed the communication establishment among the individual agents. The agents may work together to study and modify their plans, which agreed to eliminate the conflict.

\subsubsection{Knowledge-Based Negotiation (KBN)}

The basic idea of KBN:

By using the knowledge in the system science field, the conflicts are detected and resolved in the collaborative environment. The method requires the agents sharing the related knowledge field. In this way, each agent may reason and consul with other agents according to its view and others' so that the conflict would be resolute.

\subsubsection{Cost-Based Negotiation $(\mathrm{CBN})$}

G.Zlotkin and J. S.Rosenschein proposed a theoretical model and consultation agreement for negotiation. When in the non-collaborative environment, this model suppose the agents should be rational (actually they really do). Thus, the agents can still partially coordinate their 
behavior to negotiation when facing the conflict. However, the hypothesis of this model is too strict. For example: Goals are fixed for agents, each agent knows the complete knowledge. Etc. These preconditions in MAS are usually difficult to meet in practice. Based on their previous research results, a new negotiation method was proposed by adjusting the agents' initial goals. One of the key components of this new method is the cost to achieve all or part of the goal by substituting for cost.

\subsubsection{Multi-level Negotiation}

Conry in MIT proposed a multi-level negotiation agreement in 1986. The basic idea: When resource contention occurs between two agents, both sides reach an agreement as follows: One side made a concession firstly, but the other side got the priority of the resource when next conflict occurs.

\subsubsection{Network Negotiation Mechanism}

Research on the agent negotiation mechanism has been a hot topic in the study of MultiAgent System (MAS). Smith and Davis proposed the contract net protocol in the early 1980s. The protocol resolved the conflict problem between distributed resources and knowledge through the bidding process among agents. Later, more detailed research was studied based on the contract net protocol. Conry introduced multi-level negotiation into the network, allowing repeated consultation during the bidding process. Dholm used the marginal cost to help the bidding decision of the contract network. Park and Durfee studied the contract tactics, established the new model with Markov process, and determined who got the maximum effectiveness.

\section{Organizational Structure and Coordination Mechanism of Group- Subsidiary}

\subsection{Dependency Model of the Organizational Structure}

The interaction among the agents is the basic for collaboration. The dependency relation among the agents should be clear before analyzing the interaction among agents. The dependency relation based on the interaction falls into five types: Enable relations, concurrent constraint relations, shared constraint relations, prevent relations, facilitate/hinder relations.

Organizational structure is an important supporting for virtual service system in groupsubsidiary. Under the original structure, each subsidiary agent has its own targets with following the unified guidance of the group agent. Dependence relation among agents is very complex when allocating the virtual service information channel resource. The information channel resource is complex to be allocated. The relationship between each agent is more complex during the interactive process because the different dependencies in different stages. Figure 2 describes the dependence of the virtual service communications. 


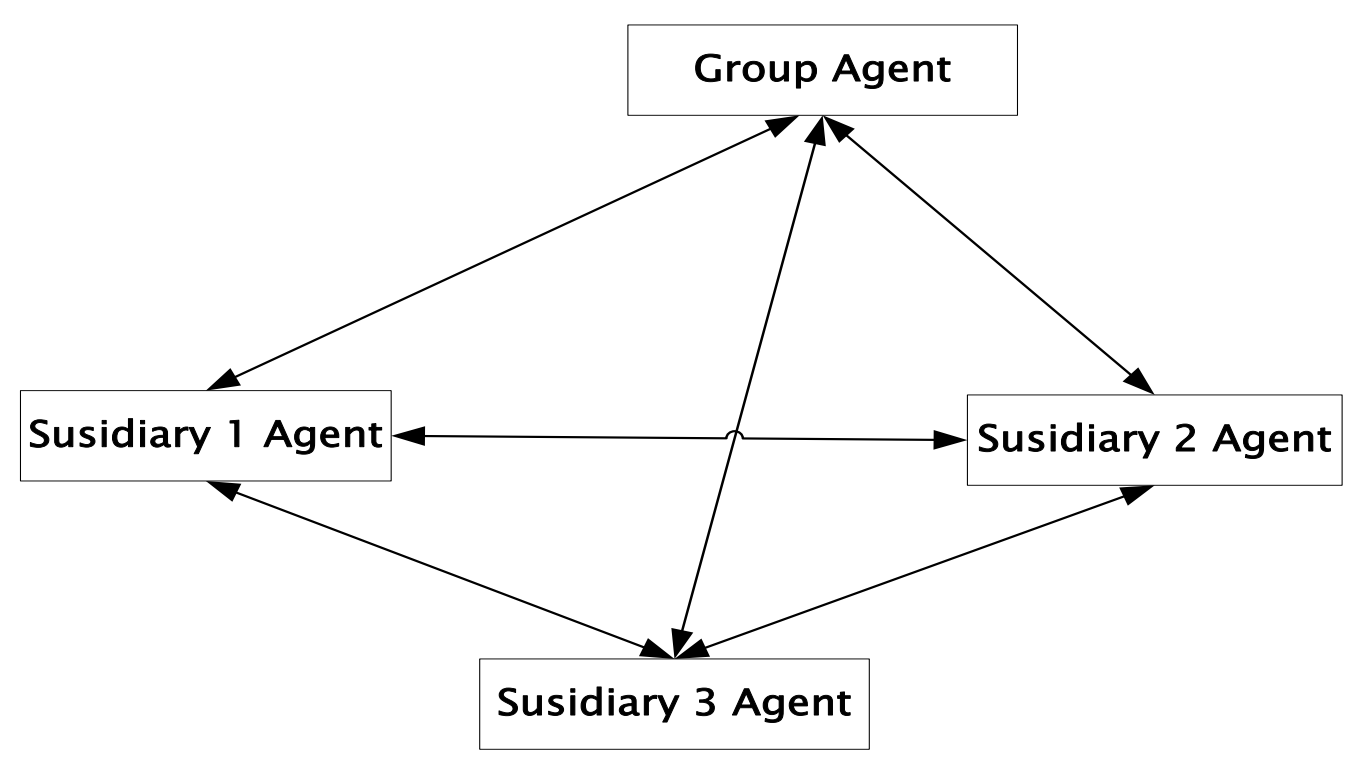

Figure 2. Model of Bulk Port Relationship Based on Organization

\subsubsection{Relationship between Group Agent and Subsidiary Agent}

The communication channel is one of the data transmission paths. The communication channel in computer network involves physical channel and the logical channel. The physical channel is the practical path for transmitting the data signal by transmission medium and the communication apparatus. The logical channel is the virtual path by data transmission of both sides based on the physical channel. The logical channel might be connected or not. Because of the network bandwidth limit and the noise signal interference, the transmission capacity is limited, which led to the problem by restricting the communication channel resources.

Communication channel resources are the public resources for virtual service system in the company. The group agent is responsible for the allocating channel resources. In the system initialization phase, each subsidiary agent submitted its actual requirements of channel resources according to their virtual service business. The group agent allocated to each subsidiary agent with the fixed channel allocation algorithm and considering the weight of the subsidiaries.

\subsubsection{Relationship among Every Subsidiary Agent}

Each subsidiary agent did not communicate with each other in the initialization phase. The submitted requirement by subsidiary agents is independence in this phase. However, in actual operation of the communication system, the channel service can't achieve completely corresponding to the requirement target. During the peak periods of communication, the channel resources were often insufficient to some subsidiary agents. The temporary idle channel should be negotiated to realize dynamic allocation between some agents. The channel shortage also should be negotiated to be allocated the urgent agent.

\subsection{Establish the Collaborative Mechanism to Break Down Tasks}

Supposing $\operatorname{Tg} p$ is the channel allocation scheme for communication group agent:

$$
T g p=\text { Collection }\left(T c p_{1}, T c p_{2}, T c p_{3} \ldots T c p_{i} \ldots T c p_{n}\right)
$$


Where $T c p_{i}$ is the submitted requirements of channel resources by subsidiary agent $i$.

$$
T c p_{i}=\operatorname{Pr} \text { ocess }\left(\operatorname{Txp}_{i, 1}, \operatorname{Txp}_{i, 2}, \operatorname{Txp}_{i, 3} \ldots \operatorname{Txp_{i,j}} \ldots \operatorname{Txp_{i,m}}\right)
$$

$T c p_{i}$ is designed according to the virtual service type, the spec of information channel, the number of involved users and other factors. Where $\operatorname{Txp}_{i, j}$ is referred respectively the corresponding indicators.

Based on the analysis above, the multi-agent cooperated with each other with the fixed mode and instructions under the organizational structure. The agent of each level is independent during the procession. The coordination work was instructed by the agent in the superior level. Therefore, the task model is composed by three dimensions: Doing agent, starting time, finishing time, just as: $T=\left\{\right.$ A gent $\left., t_{\text {start }}, t_{\text {finish }}\right\}$

The allocation scheme of the group is set as:

$$
\operatorname{Tg} p=\left\{\text { Agent }^{g}, t_{\text {start }}^{g}, t_{\text {finish }}^{g}\right\}
$$

The requirement of the subsidiary is set as:

$$
\operatorname{Tcp}_{i}=\left\{\text { Agent }_{i}^{c}, t_{i, \text { start }}^{c}, t_{i, \text { finish }}^{c}\right\}
$$

Based on the description of collaborative relation, each agent should follow the corresponding constraints during the execution of the tasks:

$$
\begin{aligned}
& t_{\text {start }}^{g} \leq \min \left(t_{i, \text { start }}^{c}\right), \quad t_{i, \text { start }}^{c}=t_{i+1, \text { start }}^{c} \\
& t_{\text {finish }}^{g} \geq \max \left(t_{i, \text { finish }}^{c}\right)
\end{aligned}
$$

(5) (6) means that the group agent must ensure to involve all the channel resource requirements of every subsidiary agent when initializing the communication process. After collecting all the requirements, the fixed channel resource with weight might be allocated to the agents. To realize the justice of each subsidiary agent, the same starting time should be guaranteed to be given.

\section{Negotiation Model Based on the Public Resource Conflict Resolution}

The collaborative model formatted by sharing the communication channel resources allocated the available channel resources to the each subsidiary agent as the arranged frequency. The advantages of this model: Each agent just assigned the corresponding channel equipment as requirement. The controlling method is simple. However, with the changing of the external environment, the relationship between each agent level is not static and immutable in the different procession. For example, when the channels of a subsidiary agent are all busy, the channels of the adjacent agent can't be used even they are idle. In this way, the channel allocation is not sufficient resulting that the channel resource utilization is low. This would occur especially when the virtual service request for channel resources concentrated sometimes. The resource request would be not achieved and the service quality would be affected. Therefore, the independent relationship between the various companies might be unable to maintain. The agents have to resolve the conflict through collaboration under the influence of the global objectives. 


\subsection{Constraint Model for Communication Channel Resources}

The channel allocation solution would affect the performance and capacity of the communication system. When multi-channel sharing, to provide more available channels for each communication terminals is the most efficient way. In reality, the channel resource must be allocated to determine the reasonable schedule for utilized adequately.

It is important to describe the communication channel as the shared resource. Under the dynamic allocation situation, the conflict resolution model that each subsidiary agent negotiated with each other to resolve the conflict is very important to resolve the conflict, rather than the model for improving the channel capacity.

The communication model was simulated as following:

The sequence $Q_{g}$ represents the required channels by all the agents asked for the virtual service.

$$
Q_{g}=q u e u e\left(Q_{c}^{1}, Q_{c}^{2}, \ldots Q_{c}^{i}, \ldots Q_{c}^{n}\right)
$$

Where $Q_{c}^{i}$ is the channel resource request by subsidiary agent i. $Q_{c}^{i}=\left\{S_{1}^{i}, S_{2}^{i} \ldots S_{j}^{i} \ldots S_{n^{i}}^{i}\right\}$

The resource request $Q_{c}^{i}$ involves the request $S_{j}^{i}$ for channel resources from 1 to n.

The allocation of the channel resources should confirm to the rule of co-frequency multiplexing according the features of the communication channels. The rule is as following: the ratio (the distance $\mathrm{D}$ among each agent using the same channel to the channel service radius $\mathrm{R}$ ) must be greater than the minimum that the channel required. As show in (8), otherwise the conflict of different services in the same virtual channel in the transmission process.

$$
\frac{D_{s_{i}-s_{i-1}}}{R_{s_{i}-s_{i-1}}} \geq f_{\text {min }}
$$

\subsection{Modeling for Public Resource Conflict Resolution Policy}

In the traditional communication system of single-level, the fixed channel allocation or dynamic channel assignment is often used to allocate the channel. The fixed channel allocation method is commonly used in the frequency allocation at present.

The dynamic channel assignment permits one channel shared by multi agents instead of fixed allocation. The channel number of each agent used is not fixed. This method would magnify the service capacity and improve the communication quality quickly. The resource was utilized more efficient. But the dynamic assignment might compute and utilize the allocation scheme every time regardless of the operation state. The response time of the channel allocation would increase clearly when the agent amount in the system is larger.

With the above analysis the two-level structure based on multi-agent was established in the operation for group-subsidiary system. The amount of subsidiary agents is fixed in the initial procession. The group allocates the channel resource to the subsidiary agents as the fixed allocation. During the operation, the channel requirement of the virtual service might appear strong fluctuations and asynchronous. The dynamic allocation method should help to deal with the assignment. The specific process is shown in Figure 3. 


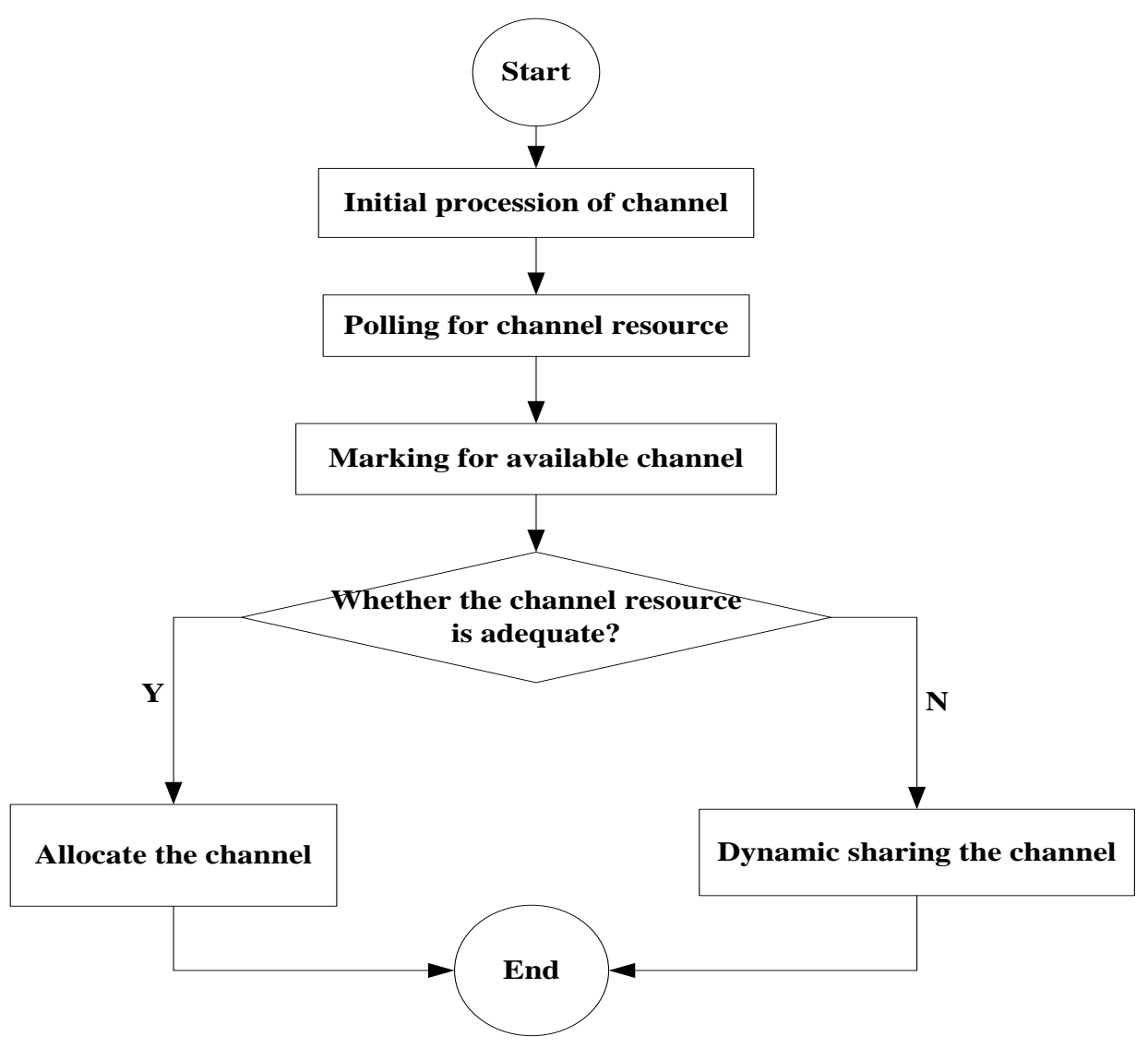

\section{Figure 3. Distribution Communication Channel Based on Conflict Resolution}

In the initial procession:

$Q_{g}=$ distribution $\left(Q_{c}^{1}, Q_{c}^{2}, \ldots Q_{c}^{i}, \ldots Q_{c}^{n}\right)$ means that after a fixed channel allocation, each subsidiary procession of the communication channel. For each subsidiary agent, $Q_{c}^{i}=\left\{\right.$ A gent $\left._{c}^{i}, f_{\text {total }}^{i}, f_{\text {rest }}{ }^{i}\right\}$, where $Q_{c}^{i}$ is channel state of the subsidiary agent. A gent ${ }_{c}^{i}$ represents the corresponding agent. ${ }^{f_{\text {total }}^{i}}$ is the total amount for allocated channel. $f_{\text {rest }}^{i}$ is the residual amount of allocated channel.

In the operation procession:

The channel requirement $R_{j, k}^{i}$ by the virtual service $S_{j}^{i}$

$$
\begin{aligned}
& R_{j, k}^{i}=\left\{Q_{c}^{i}, S_{j}^{i}, P n_{j, k}^{i}, f_{\text {request } j, k}^{i}, t_{\text {start } j, k}^{i}\right\} \\
& \text { If } f_{\text {request } j, k}^{i} \leq f_{\text {rest }}^{i}
\end{aligned}
$$

The $A g$ ent $t_{c}^{i}$ allocated the resource by itself.

Else

The requirement was added to the dynamic set $A g e n t_{c}^{i}$ to be assigned. 
When polling and get the prerequisite $U_{c f l} \neq \varphi$, the set $U_{s p l}=\left\{Q_{c}^{1}, \ldots Q_{c}^{x}\right\}$ should be selected because it matched the rule of co-frequency multiplexing. Based on this selection method, the corresponding channels could be allocated to resolve the conflict.

For the set of virtual service requirement $U_{c f l}$, it is suppose that the resource conflict existed between $R_{j, x}^{i}$ and $R_{j, y}^{i}$. Though $R_{j, x}^{i}$ and $R_{j, y}^{i}$ have the description about the time request $t_{\text {start } j, x}^{i}$ and $t_{\text {start } j, y}^{i}$, the actual application request must be considered, and it is necessary to establish the corresponding request priority queue method for resolve the resource conflict.

In the set $R_{j, k}^{i}=\left\{Q_{c}^{i}, S_{j}^{i}, P n_{j, k}^{i}, f_{\text {request } j, k}^{i}, t_{\text {start } j, k}^{i}\right\}, S_{j}^{i}$ represents the priority level of the virtual service. $P n_{j, k}^{i}$ is the k-th request amount. $f_{\text {request } j, k}^{i}$ is the channel amount of this request. In order to facilitate comparison of the request, the model was established as following to compare the request conflicts. With the priority comparing result of $\operatorname{conflict} R_{j, k}^{i}$, the request queue was reordered.

$$
V_{j, k}^{i}=\left\{S_{j}^{i}, P n_{j, k}^{i}, f_{\text {request } j, k}^{i}, t_{\text {start } j, k}^{i}\right\}
$$

Reorder conflict queue is an important step to achieve a coordinated sharing of channel resource. The conflict collaboration model includes two aspects: (1) to establish the decisionmaking strategies to ensure all participated agents agree with the solution. The agents have the ability to confirm the negotiation result. (2) To ensure the agents have the ability to decide next actions through the negotiation. As shown in Table 1, the agents should follow the decision rules when decide next actions.

\section{Table 1. Description of Agent's decision rules based on Dynamic Channel} Distribution

\begin{tabular}{|c|c|c|}
\hline Se. & Rules & Description \\
\hline 1 & $\operatorname{csp}$ rule & $\begin{array}{l}\text { Compare compensation parameters } c c p t_{i} \text { corresponding to } R_{j, k}^{i} \text { in the } \\
\operatorname{conflict~set~} U_{c f l} \text {, Select } R_{j, k}^{i} \text { to } \max \left(c c p t_{i}\right)\end{array}$ \\
\hline 2 & $S$ rule & $\begin{array}{l}\text { Compare virtual service level } S_{j} \text { corresponding to } R_{j, k}^{i} \text { in the conflict } \\
\operatorname{set} U_{c f l} \text {, Select } S_{j}^{i} \text { to } \max \left(S_{j}\right)\end{array}$ \\
\hline 3 & $P n$ rule & $\begin{array}{l}\text { Compare service amount } P n_{j} \text { corresponding to } R_{j, k}^{i} \text { in the conflict } \\
\operatorname{set} U_{c f l} \text {, Select } R_{j, k}^{i} \text { to } \max \left(P n_{j}\right)\end{array}$ \\
\hline 4 & $F$ rule & $\begin{array}{l}\text { Compare request amount for channel } F_{j} \text { corresponding to } R_{j, k}^{i} \text { in the } \\
\operatorname{conflict} \operatorname{set} U_{c f l}, \text { Select } R_{j, k}^{i} \text { to } \max \left(F_{j}\right)\end{array}$ \\
\hline 5 & $T$ rule & $\begin{array}{l}\text { Compare starting time of request } F_{j} \text { corresponding to } R_{j, k}^{i} \text { in the } \\
\operatorname{conflict~} \operatorname{set} U_{c f l}, \text { Select } R_{j, k}^{i} \text { to } \max \left(T_{j}\right)\end{array}$ \\
\hline
\end{tabular}




$$
\text { ccpt } \succ S \succ P n \succ F \succ T
$$

(10) shows the rule priority when participating consultation decision-making. Where $c c p t_{j, k}^{i}$ the compensation parameter for the conflict requirement is $R_{j, k}^{i}$, represents the accumulated compensation when $R_{j, k}^{i}$ failed to get the conflict queue. So $c c p t_{i} \geq 0$.

Based on the dynamic compensation, the negotiation strategy model was established to realize the negotiation conflict resolution. This model aimed at resolve the conflict starting from the justice in the procession. The rules from Tab1-rule2, 3, 4, 5 can be seen on request sorting criteria more virtual services from its channel service. These rules project the value of the virtual service contrasted to the fixed channel allocation method. The basic idea of the negotiation strategy model based on the dynamic compensation is: With introducing the compensation parameters, the agents can be consulted from some of the last round negotiation to determine next plan to get positive feedback, so that this round negotiation would guarantee more balanced for the parameters.

\section{Conclusion}

The features of large, complex and service virtualization in the information network corporation were analyzed in this paper. Based on the organizational structure, the coordination mechanism was designed to describe the collaborative relationship between the group and subsidiaries. Based on the public resource conflict resolution, the negotiation model was established to realize the coexistence of the fixed allocation and the dynamic allocation. With the introduction of dynamic compensation, the channel resource conflict might be resolved quickly and the virtual service would be finished successfully.

\section{References}

[1] S. Kraus, J. Wilkenfeld and » G. » Zlotkin, "Multi agent negotiation under time constraints", Artificial Intelligence, vol. 75, (1995), pp. 297-345.

[2] S. E. Conry, K. Kuwabara, V. R. Lesser and R. A. Meyer, "Multistage negotiation for distributed constraint satisfaction", IEEE Transactions on Systems, Man and Cybernetics, vol. 21, no. 6, (1992).

[3] S. Buyun, F. Zhanfeng and Y. Minzhong, "Conflict resolution of CAPP based on multi-agent system", Modular Machine Tool \& Automatic Manufacturing Technique, vol. 10, (2001).

[4] W. Baogang, "Conflict resolution research based on negotiation", Journal of Chinese Computer Systems, vol. $11,(\mathbf{1 9 9 8})$.

[5] W. Baogang and P. Yunhe, "Research on the cooperation design technology", China Mechanical Engineering, vol. 4, (1999).

[6] R. Korobkin, "Negotiation: Theory and Strategy", ASPEN, vol. 2, (2009).

[7] W. Zhongbin, "Research on a Type of Cooperative CAPP System", Nanjing University of Aeronautics and Astronautics doctor's dissertation, vol. 5, (2002).

[8] N. R. Jennings and E. H., "Using joint responsibility to coordinate collaborative problem solving in dynamic environments", In Proceedings the Tenth National Conference on Artificial Intelligence, (1992). 